\section{Commentary: Central venous access in congenital heart surgery: Is it time for surgeons to toe (and place) the line?}

\author{
Jennifer I. Sherwin, MD, \\ Veerajalandhar Allareddy, MD, and \\ Nicholas D. Andersen, MD
}

Most pediatric cardiac care providers can recall an infant who decompensated rapidly and because they were very close to discharge had no reliable intravenous access. Resuscitation efforts are especially challenging without the ability to deliver medications rapidly and reliably. A dramatic example, but it highlights the importance of dependable, safe, long-term central access for a certain subset of patients with high-risk congenital cardiac conditions. Although no intensivist would deny the desire for ample access in a freshly postoperative patient, the more difficult question lies in the appropriate type and duration of central access as these patients convalesce.

Ghani and colleagues ${ }^{1}$ suggest a novel and innovative approach to central venous access placement in children undergoing heart surgery. Specifically, they present a cost analysis of transdiaphragmatic tunneled Broviac catheters (TBCs) placed intraoperatively, compared with the more conventional long-term central access option, the peripherally inserted central catheter (PICC). A transdiaphragmatic TBC is inserted at the beginning of an operation and is tunneled through the diaphragmatic pericardium to the junction of the right atrium and inferior vena cava. This technique ensures a short intravascular course and prevents migration beyond the right atrium. The pericardial space is avoided allowing the line to be safely removed at the

\footnotetext{
From Division of Cardiovascular and Thoracic Surgery, Duke University Medical Center, Durham, NC.

Disclosures: The authors reported no conflicts of interest.

The Journal policy requires editors and reviewers to disclose conflicts of interest and to decline handling or reviewing manuscripts for which they may have a conflict of interest. The editors and reviewers of this article have no conflicts of interest.

Received for publication April 20, 2020; accepted for publication April 21, 2020; available ahead of print May 1, 2020.

Address for reprints: Nicholas D. Andersen, MD, Division of Cardiovascular and Thoracic Surgery, Duke University Medical Center, 2301 Erwin Rd, DUMC 3474, Durham, NC 27710 (E-mail: Nicholas.Andersen@Duke.edu).

J Thorac Cardiovasc Surg 2020;160:1567-8

0022-5223/\$36.00

Copyright (C) 2020 by The American Association for Thoracic Surgery

http://dx.doi.org/10.1016/j.jtcvs.2020.04.066
}

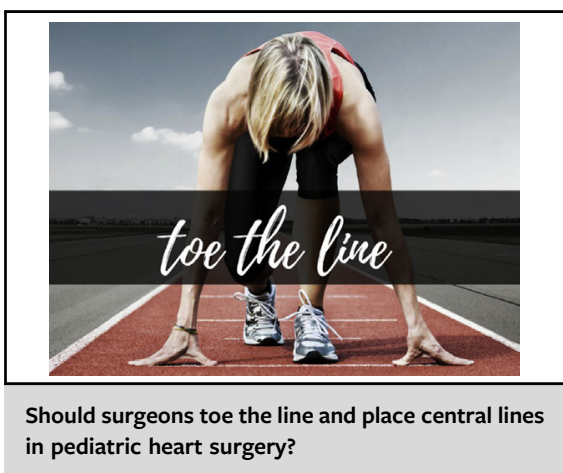

CENTRAL MESSAGE

The surgeon-placed transdiaphragmatic tunneled Broviac catheter is an innovative and less expensive alternative to peripherally inserted central catheters in infants undergoing cardiac surgery.

bedside, outside of the intensive care unit, unlike a transthoracic line.

This innovative method is a welcome idea, with potential for balancing the need for long-term access with an acceptable safety profile, thereby increasing provider comfort with leaving a central line in place until discharge. The current study focuses on the economics of the transdiaphragmatic TBC compared with PICC. Although interesting, this represents only the tip of the iceberg regarding the value of this type of line. In general, these TBCs are large enough to have blood drawn from them. This is a major advantage over PICC lines in smaller patients where either the line will not draw or risk of thrombus is greatly increased by this exercise. Although, PICCs are preferred over other large central vein catheters in a convalescing child, they are not trivial procedures and are associated with significant morbidity. ${ }^{2}$

The apparent benefits of the transdiaphragmatic TBC are very encouraging, but before its more widespread adoption, a full assessment of countermeasures is necessary. The current analysis looks at complications related to removal and dislodgement of TBCs and transthoracic lines, but not other complications associated with central lines such as thrombus and infection. It is also not entirely clear if there is a learning curve to this approach, and how many liver capsule hematomas or ex-laps for hemoperitoneum will result from the novice operator attempting to replicate this technique. But, if the transdiaphragmatic TBC is found to 


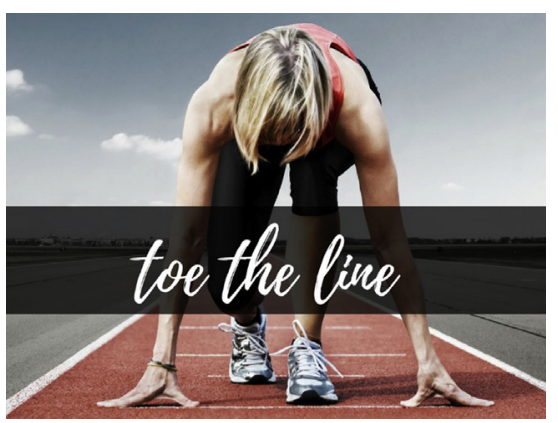

FIGURE 1. Should surgeons toe the line and place central lines in pediatric heart surgery?

be less expensive and associated with fewer complications, this would prove its superiority to a PICC and suggest that it is time for surgeons to toe the line (Figure 1) and place more central lines at the time of surgery.
We cannot forget the importance of PICC lines in the group of patients who require central access before surgery. The comparison of the safety profiles of the TBC and PICC would be useful in determining whether an already existing PICC should be traded out for a TBC if and when the patient goes to the operating room. Regardless, providers will appreciate the effort by surgeons to seek alternative methods of safe, reliable access for a high-risk patient population to improve the ability to deliver efficient, optimal care throughout the hospital stay.

\section{References}

1. Ghani MO, Raees MA, Tang AR, Anandan D, Shannon CN, Bichell DP. Transdiaphragmatic tunneled Broviac catheters: cost-effective perioperative central venous access in infants undergoing cardiac surgery. J Thorac Cardiovasc Surg. 2020; 160:1559-66.

2. Badheka A, Bloxham J, Schmitz A, Freyenberger B, Wang T, Rampa S, et al. Outcomes associated with peripherally inserted central catheters in hospitalised children: a retrospective 7-year single-centre experience. BMJ Open. 2019;9: $\mathrm{e} 026031$.
See Article page 1559.

\section{Commentary: \\ Transdiaphragmatic tunneled \\ Broviac catheters: How small ripples can make big waves}

\author{
Minoo N. Kavarana, MD, FACS
}

Ghani and colleagues ${ }^{1}$ describe a retrospective cost analysis comparing a novel technique of transdiaphragmatic tunneled Broviac catheter (TBC) placement into the right atrium (RA) compared with traditional percutaneous indwelling central catheter (PICC) placement in infants undergoing congenital

\footnotetext{
From the Section of Pediatric Cardiothoracic Surgery, Medical University of South Carolina, Charleston, SC.

Disclosures: The author reported no conflicts of interest.

The Journal policy requires editors and reviewers to disclose conflicts of interest and to decline handling or reviewing manuscripts for which they may have a conflict of interest. The editors and reviewers of this article have no conflicts of interest.

Received for publication April 24, 2020; accepted for publication April 25, 2020; available ahead of print May 1, 2020

Address for reprints: Minoo N. Kavarana, MD, FACS, Section of Pediatric Cardiothoracic Surgery, Medical University of South Carolina, 10 McClennan Banks Dr, SJ 2190 N/MSC 918, Charleston, SC 29425 (E-mail: kavarana@musc.edu).

J Thorac Cardiovasc Surg 2020;160:1568-9

$0022-5223 / \$ 36.00$

Copyright $(2) 2020$ by The American Association for Thoracic Surgery

http://dx.doi.org/10.1016/j.jtcvs.2020.04.067
}

Check for updates

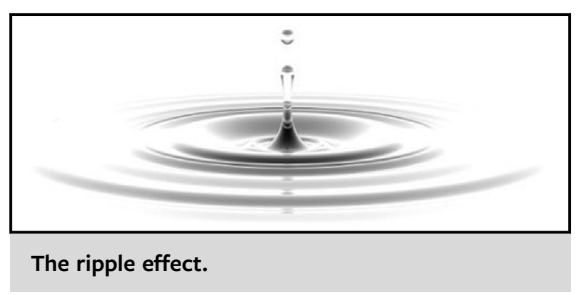

CENTRAL MESSAGE

Transdiaphragmatic tunneled

Broviac catheters provide a costeffective, reliable, and durable method for perioperative central venous access during pediatric cardiac surgery.

heart surgery. In their study, they propensity matched the patients with and without TBCs to compare the costs of the TBC and non-TBC perioperative line strategies. Eighty-three patients received $\mathrm{TBC}$ and 137 did not receive TBC, and the final propensity-matched cohort included 82 patients in 